\begin{tabular}{|c|c|c|c|c|}
\hline 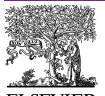 & Opinion & TRENDS in Cognitive Sciences & Vol.8 No.5 May 2004 & $\begin{array}{l}\text { Full text provided by www.sciencedirect.com } \\
\text { science doirect. }\end{array}$ \\
\hline
\end{tabular}

\title{
Mining event-related brain dynamics
}

\author{
Scott Makeig ${ }^{1}$, Stefan Debener ${ }^{2}$, Julie Onton ${ }^{1}$ and Arnaud Delorme ${ }^{1}$ \\ ${ }^{1}$ Swartz Center for Computational Neuroscience, Institute for Neural Computation, University of California San Diego, \\ La Jolla CA 92093-0961, USA \\ ${ }^{2}$ University of Hamburg, Hamburg, Germany
}

\begin{abstract}
This article provides a new, more comprehensive view of event-related brain dynamics founded on an information-based approach to modeling electroencephalographic (EEG) dynamics. Most EEG research focuses either on peaks 'evoked' in average eventrelated potentials (ERPs) or on changes 'induced' in the EEG power spectrum by experimental events. Although these measures are nearly complementary, they do not fully model the event-related dynamics in the data, and cannot isolate the signals of the contributing cortical areas. We propose that many ERPs and other EEG features are better viewed as time/frequency perturbations of underlying field potential processes. The new approach combines independent component analysis (ICA), time/frequency analysis, and trial-by-trial visualization that measures EEG source dynamics without requiring an explicit head model.
\end{abstract}

Scalp EEG signals are produced by partial synchronization of neuronal-scale field potentials across areas of cortex of centimetre-squared scale. Although once viewed by some as a form of brain 'noise', it appears increasingly probable that this synchronization optimizes relations between spike-mediated 'top-down' and 'bottom-up' communication, both within and between brain areas. This optimization might have particular importance during motivated anticipation of, and attention to, meaningful events and associations - and in response to their anticipated consequences [1-3]. This new view of cortical and scalp-recorded field dynamics requires a new data analysis approach. Here, we suggest how a combination of signal processing and visualization methods can give a more adequate model of the spatially distributed eventrelated EEG dynamics that support cognitive events.

Traditional analysis of event-related EEG data proceeds in one of two directions. In the time-domain approach, researchers average a set of data trials or epochs time-locked to some class of events, yielding an ERP waveform at each data channel. The frequency-domain approach averages changes in the frequency power spectrum of the whole EEG data time locked to the same events, producing a two-dimensional image that we call the eventrelated spectral perturbation (ERSP; see Box 1).

Neither ERP nor ERSP measures of event-related data fully model their dynamics. Imagine, by analogy, a snapshot of a seashore view created by averaging together a large number of snapshots taken at different times. This average snapshot would not show the waves! Similarly, ERP averaging filters out most of the EEG data, leaving only a small portion phase-locked to the time-locking events (see Box 1). The ERP and ERSP are nearly complementary. Oscillatory (ERSP) changes 'induced' by experimental events can be poorly represented in, or completely absent from the time-domain features of the ERP 'evoked' by the same events [4], and vice versa. But even together, the ERP and ERSP do not fully capture the

\section{Box 1. Concepts and terms}

EEG phase: The phase of an EEG wave train or packet at a best-fitting frequency is determined by the latencies of its features relative to a reference event. For example, a wave in sine phase at the reference event is positive-going, whereas a wave at cosine phase reaches its positive peak there, etc. Phase is a circular measure.

EEG phase resetting: Imagine a set of data trials time locked to unexpected events, and a selected analysis frequency. Prior to the events, the distribution of EEG phase across trials should be uniform around the phase circle. Following the events, however, the EEG phase distribution can become lopsided. If it now has only one value, the signal has become completely 'phase-locked' to the events. This imbalance could be produced by the simple addition of an ERP of fixed polarity (and phase) to each trial, or by transient perturbation of the phase of synchronized local field activity within cortical EEG source areas. Such non-linear 'phase resetting' occurs in many dynamic and biological model systems [20]. A well-known example is the shifting of the phase of the sleep-wake schedule by earlymorning exposure to bright light.

ERP: event-related potential, a 1-D time series of mean potential deviations (in $\mu \mathrm{V}$ ) from baseline.

ERP image: a 2-D (trial-by-latency) image of the color-coded trial waveforms sorted in order of some variable of interest (see Figure 2). ERSP: event-related spectral perturbation, a 2-D (frequency-bylatency) image of mean change in spectral power (in $d B$ ) from baseline (see Figure 1).

ICA: independent component analysis, a statistical linear decomposition of the data into a sum of components with fixed scalp maps, such that each of the components contributes as much distinct information to the data as possible. ICA methods were introduced in France in the early 1990s $[47,48]$. First applications followed to EEG $[25,28]$, MEG $[34,49,50]$ and many other types of data [26]. Infomax ICA $[28,29]$ first transforms the data non-linearly to spread it out, then adjusts the single-channel data axes gradually to make the data points still more evenly distributed, like molecules of gas in a box. The data components specified by the final axis directions are maximally independent.

ITC: inter-trial coherence, a 2-D (frequency-by-latency) image of the strength ( 0 to 1$)$ of phase locking of the EEG signals to the timelocking events (see Figure 1).

PCA: principal component analysis, a deterministic linear decomposition of the data into a sum of components with fixed orthogonal scalp maps, such that each successive component accounts for as much of the remaining data variance as possible. 
mean event-related dynamics expressed in the data. For example, event-related changes in the distribution of oscillatory EEG phase (see Box 1) produce ERP features [5], but ERPs do not indicate the strength of this phaselocking in the EEG data.

\section{EEG sources}

Basic facts about cortical physiology allow some predictions about the cortical sources of EEG signals. Cortex has a very high density of local connections [6] and is electrically far from the scalp, so sources of EEG signals are most probably compact regions of cortex whose local field activities at the neuronal scale are similarly oriented by cortical geometry and partially synchronized at the $\mathrm{cm}^{2}$-scale, thereby producing far-field EEG signals that reach the scalp by volume conduction [7]. Although a simple model (for alternatives, see [8-11]), this concept of an EEG source is generally compatible with cortical physiology $[9,12,13]$ and with the EEG results we describe below.

Every EEG measure best fits a model of EEG dynamics that might be called its associated 'brain view'.

\section{The ERP brain view}

ERPs were originally associated with a sequential, 'bottom-up' view of brain processing of sensory stimulus events. In this view, the signs of this processing were considered to be the reliable sequence of brief, monophasic 'evoked' potential peaks captured in sensory-evoked ERPs, plus 'random background' EEG processes whose phase distributions were independent of, and totally unaffected by experimental events. Recent neurophysiological evidence, however, strongly supports the importance of early and concurrent 'top-down' and 'bottom-up' signaling during active perception, even in primary sensory cortex $[14,15]$. The relative temporal 'compactness' of cortex means that activity in several source areas can contribute to each ERP feature later than a few tens of ms after stimulus onsets. Each cortical source can also contribute to more than one ERP peak [15].

\section{Is the brain baseline flat?}

Contrary to the typically flat appearance of the prestimulus ERP baseline, EEG signals (and the cortex itself) are typically neither silent nor disengaged before experimental events. Post-stimulus increases in the relative proportion of positive or negative EEG potential values, across trials, produce ERP features - independent of whether or not EEG signal energy is increasing or decreasing $[5,16]$. This is again contrary to the visual impression given by ERPs that 'stimulus-evoked' activity arises from a static activity baseline.

\section{Problem of definition}

In essence, averaging EEG data trials collapses their rich event-related dynamics into a single statistical measure, the ERP, discarding in the process much of the dynamic information contained in the original data. At root, although contrary to usual assumption, the ERP average is not defined in single trials, and any ERP feature might be absent from, or appear differently in, any or all of the single trials used to compute it. This apparent disconnect between ERPs and the EEG data from which they are derived has separated ERP from other EEG research for decades.

\section{The ERSP brain view}

Here, the EEG data recorded at each scalp channel and frequency are considered to be ongoing EEG rhythmic activity whose frequency power spectrum is transiently modulated or perturbed, for example by event-related acetylcholine, norepinephrine and/or serotonin signals $[17,18]$. However, the activities of the cortical EEG sources can be concurrently modulated by more than one such process, and again each scalp electrode records a weighted mixture of different cortical sources. Thus, a single peak or valley in an ERSP image might index the combined effects of multiple processes modulating the activities of several EEG sources that project to the recording electrode. Also, EEG polarity, positive or negative, does matter [19], but the ERSP ignores it. In particular, the ERSP brain view ignores the possibility of event-related phase resetting (see Box 1) of ongoing activity by experimental events [20].

Both these 'brain views' ignore two important facts: (1) Spatial mixing

The accurate interpretation of ERP and ERSP measures is strongly compromised by signal mixing at the electrodes produced by volume conduction. Each scalp electrode indiscriminately records the sum of all the potentials conducted to it from both cortical and non-brain sources (eyes, muscle, etc.). When, as is often the case, the different source potentials arriving at the same electrode have different signs, they cancel rather than sum and thus are not accurately represented in the ERP and ERSP or in the raw EEG.

(2) Phase resetting

Following unexpected experimental events, the expected uniform random distribution, across trials, of the phase of the EEG activity at one or more frequencies can partially collapse. Such partial 'phase resetting' induced by events contributes to the ERP without any increase in EEG power. For example, prolonged posterior 'alpha-ringing' can appear in a visual stimulus ERP through phase resetting of central occipital alpha activity [5]. However, the similarities between ERP potential maps of and EEG power maps [5], as well as many results of the analysis approach we outline below, suggest that partial phaseresetting of ongoing EEG processes contributes to many ERP features.

\section{New models, new measures}

A novel approach to EEG (and/or, equally, to magnetoencephalographic or MEG) data analysis applies trial-bytrial visualization and time/frequency analysis to the extracted activities of cortical EEG sources that are first identified and spatially separated not on the basis of ERP or ERSP scalp distributions, but by the unique temporal information they contribute to the whole recorded signals. Brain dynamic responses to events are then modeled as transient perturbations of the ongoing EEG activities of these sources. 


\section{Independent component analysis}

Spatial filters that highlight spatially coherent local field activity at a given brain location can be constructed for high-density MEG [21-23] and EEG [7] data. However, to separate the recorded data into functionally distinct local field processes, one must either know the locations of the cortical patches supporting them or else determine these locations by the distinctiveness of their activities. Directly inverting the recorded scalp field maps are not the answer because any such map can be generated by a large number of different multi-source models. When the number of active sources is quite small, biophysical inverse methods can successfully separate them by identifying them as global or local directions of maximum variance in the data [24]. Unfortunately, however, even the severe data reduction produced by ERP averaging might not sufficiently reduce the number of cortical sources contributing to the data.

A radically different approach begins by applying independent component analysis (ICA) to the concatenated single trial data $[5,25,26]$. 'Linear data decompositions', which include ICA, principal component analysis (PCA; see Box 1) and its derivatives, separate multichannel data into a sum of activities of components each comprised of a time course of its activity in every trial and a single scalp map giving the strength of the volumeconducted component activity at each scalp electrode. A linear ICA decomposition is one whose component activities are most temporally independent in some sense [27], and therefore also most temporally 'distinct'. Given adequate training data, runica, an automated form [28] of the extended infomax ICA algorithm [27,29], can identify the ICA components of the data concurrently with high efficiency [30], even from hundreds of data channels.

\section{Information versus variance}

Every EEG electrode montage acts as a set of spatial filters of cortical field dynamics. ICA simply performs further linear spatial filtering on the recorded data to cancel the effects of summing of the volume-conducted cortical source activities in each of the recording channels. ICA identifies sources by finding distinctive sources of information in the data. If the activity time courses of different EEG components are relatively independent of one another, their local field activities must be largely decoupled physiologically. Thus, under favorable circumstances ICA should separate EEG (or equally MEG) data into physiologically and functionally distinct sources.

Results of actual data decompositions demonstrate that ICA, applied to sufficiently large and clean high-density EEG datasets, can separate out the large or small activities, scalp maps, and scalp data contributions of dozens of maximally independent information sources whose scalp maps fit near-perfectly the dipolar projections of cortical EEG sources [31-33] (see Figure 1). At the same time, ICA separates the contributions of stereotyped nonbrain artifact signals including eye movements, line noise, and muscle activities [34]. Applying runica to infomax ICA (as implemented in [35]) to 71-channel data, we currently recover nearly 20 maximally independent components whose scalp maps reasonably resemble the projection of a single dipole (or in some cases a bilaterally symmetric dipole pair) [36] (Figure 2). Residual scalp map variance for the best-fitting single- or dual-dipole model is often surprisingly low (Figures 1,2) given the relative inaccuracy of the spherical head model we use to compute it.

\section{ICA advantages}

Unlike PCA, ICA does not require the scalp maps as well as the time courses of the components to be orthogonal [37]. Unlike Laplacian sharpening filters [38], ICA separates EEG sources with tangential as well as radial orientations (Figure 1). That ICA decomposes the data into sources with independent time courses and dipolar scalp maps, without taking into account any information about head geometry or electrode locations, strongly suggests that the recovered components index physiologically distinct processes. This result might surprise researchers accustomed to thinking that EEG sources can only be separated by directly solving the biophysical inverse problem, which is heavily underdetermined even for moderate numbers of sources [24]. Our comparisons of independent components collected during performance of the same task across subjects (based on scalp maps, power spectra, and/or other activity measures), have revealed common groups of EEG processes with distinct spatial and event-related dynamic characteristics that together account for most of the nonartifactual EEG. Not surprisingly, these component clusters have much in common with traditionally recognized types of EEG activity [5], albeit with enhanced spatial definition and signal-to-noise ratio relative to the single scalp electrode data [39].

\section{Time/frequency analysis}

Analysis of event-related changes in spectral power and phase consistency across single trials time locked to experimental events can characterize event-related perturbations in the oscillatory dynamics of ongoing EEG signals. Applying such measures to the activity time courses of separated independent component sources avoids the confounds caused by positive and negative potentials from different sources canceling each other at the recording electrodes, and by misallocating to the recording electrodes activity that originates in various, often distant, cortical sources. We use the ERSP [4] to measure event-locked changes in spectral power, and inter-trial phase coherence (ITC, first introduced as 'phase-locking factor' [40]; see Box 1), to measure the appearance and degree of this consistency near experimental events. From the frequency-domain point of view, ERPs are produced by this phase locking. Thus, ITC images decompose the ERP into its constituent phaselocked frequency bands (Figure 1), but provide additional information about the relationship between the ERP and whole EEG data.

\section{An event-related time/frequency state space}

Considered together, the ERSP and ITC provide a conceptual framework for observing and modeling the event-related brain dynamics that occur consistently across a set of trials. The two measures can be said to 


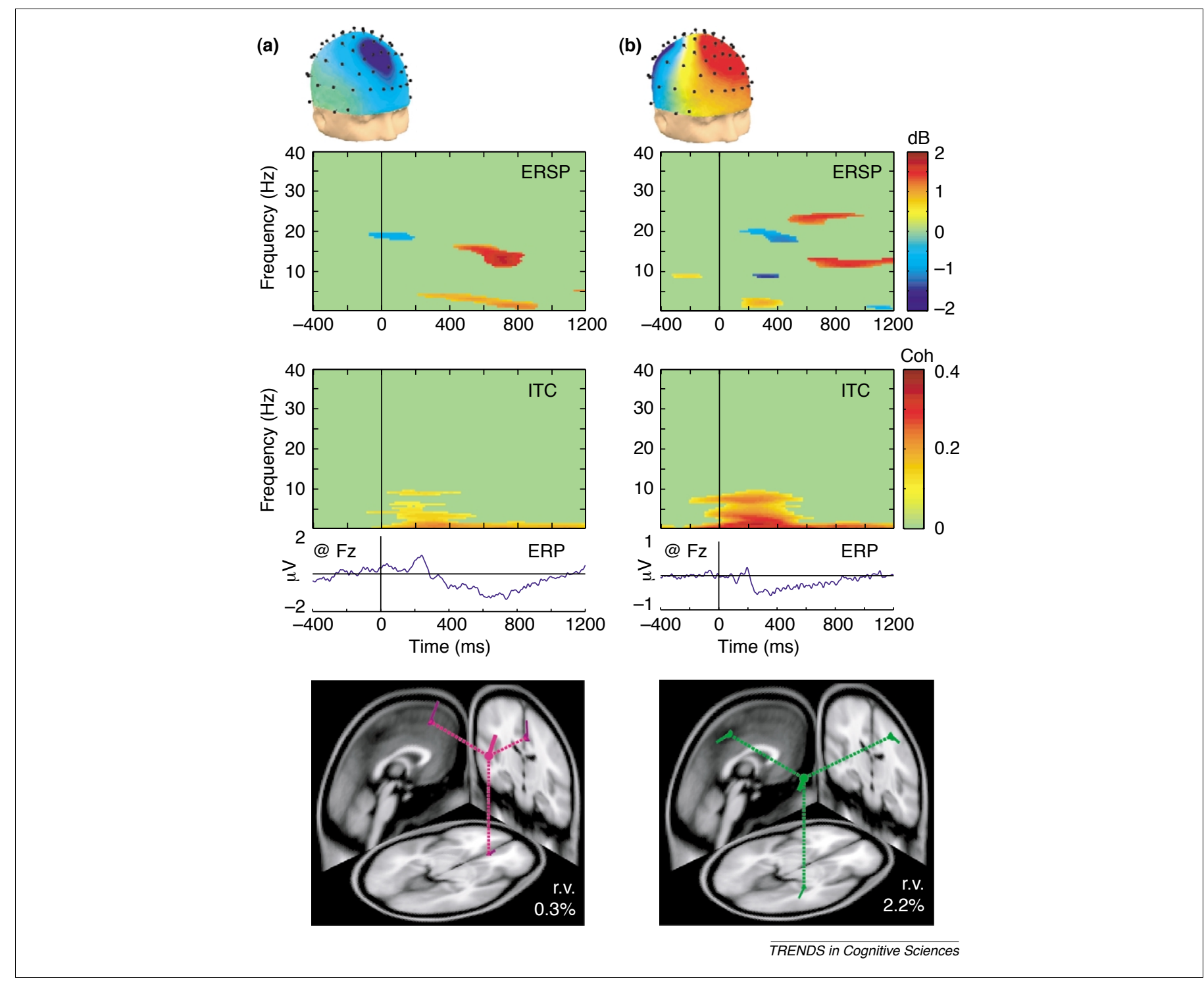

Figure 1. Maximally independent EEG components compatible with a single cortical source. Scalp maps, ERSP and ITC images, and ERPs for two typical independent EEG processes during visual letter encoding (green in images indicates $\mathrm{p}>0.001$ ) [35]. Below are the best-fitting equivalent current dipole (ECD) models ('r.v.' is the residual scalp map variance across the 69 scalp electrodes). (a) Frontal midline theta component with radial ECD. Spectral power increases shown in the ERSP accompany weak phase locking on ITC and slow ERP effects ( $\mu \mathrm{V}$ as at Fz). (b) Mu-rhythm component with tangential ECD in right somatomotor cortex. Alpha- and beta-band (ERSP) power changes, accompanied by partial phase locking (ITC) contributing to the component ERP.

form a 'time/frequency state space' that, at each trial latency, has dimensions: (1) EEG frequency, (2) EEG power relative to baseline (measured by ERSP), and (3) absolute level of phase consistency (measured by ITC) (see Figure 3). The spectral state-space model emphasizes that event-related EEG phenomena generally have both 'evoked' and 'induced' aspects measured by ITC and ERSP respectively. Figure 3 shows state-space locations indicating ('pure') narrow-band power decreases and increases (event-related [local] desynchronization, ERD, and synchronization, ERS, respectively [41]), and prototypic ERP and partial phase-resetting (PPR) features. However, every region in the space (e.g. '?' in Figure 3) represents an observable feature of event-related data. As an example, the quite different trial-by-trial dynamics of two bilaterally near-symmetric posterior independent component processes are shown in Figure 2.

\section{ERP-image visualization}

ERP image plots are useful for visualizing trial-by-trial consistencies in a set of event-related data trials [31,34]. Essentially, 2-D ERP images generalize 1-D ERP averaging. Instead of simply summing all the data trials, trials are first sorted in order of a relevant data or external variable, optionally smoothed across neighboring trials in the sorting order, and then plotted as a color-coded twodimensional image (Figure 2). ERP image plots constructed using an appropriate trial-sorting variable often reveal trial-to-trial consistencies otherwise hidden in total EEG variability, particularly when applied to the activities of ICA sources. ERP image plots reveal that variability across trials in the scalp data are usually the sum of distinct and more constrained trial-to-trial variabilities in the contributing independent source activities (as in Figure 2). For example, trials sorted by subject reaction 


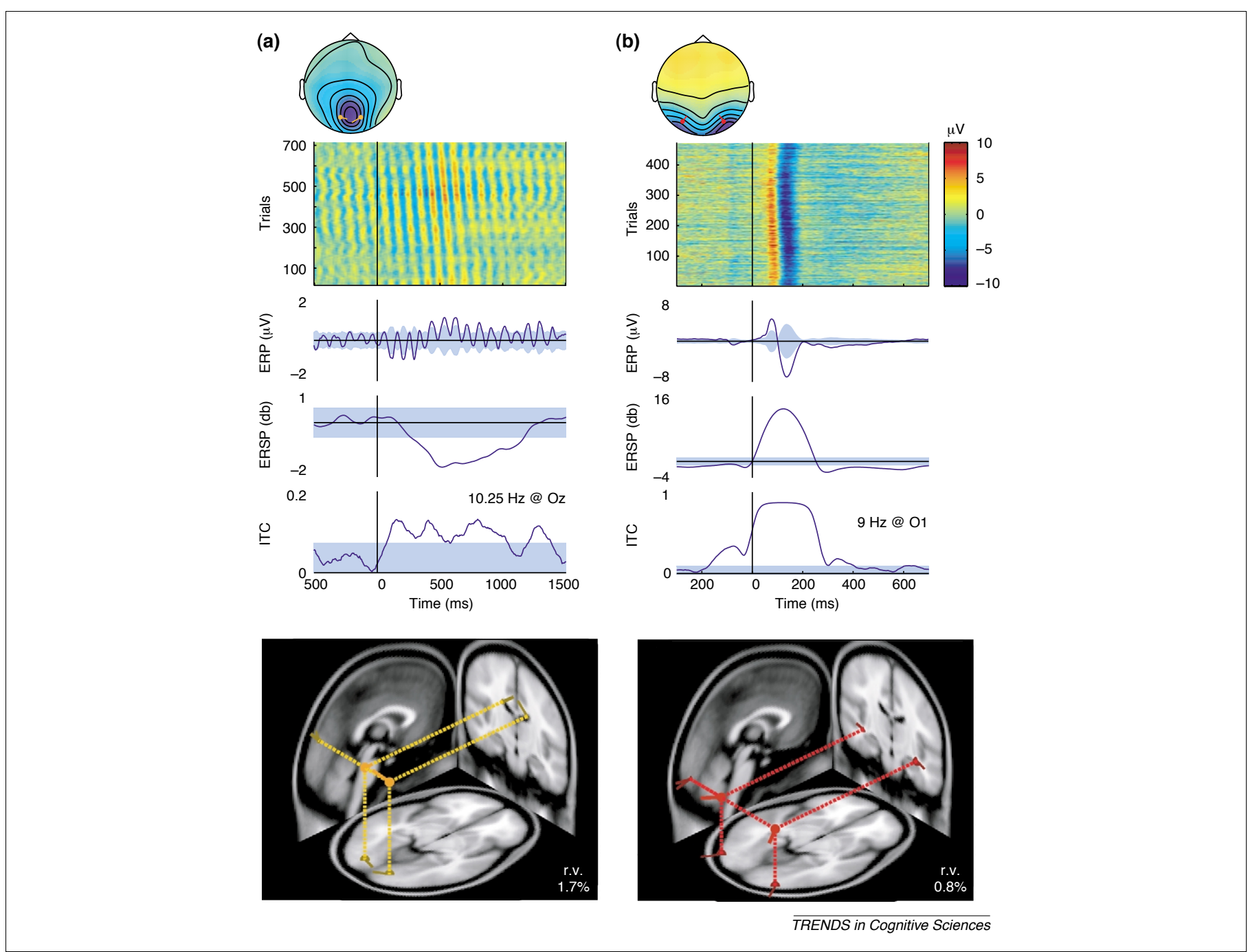

Figure 2. Independent EEG components with distinct event-related dynamics. (a) Bilateral component showing weak alpha phase resetting during partial alpha blocking. From infomax decomposition of 70031 -channel trials during visual-spatial selective attention [5]. Top: component scalp map (as in Figure 1). Center: ERP image showing color-coded single trials sorted by alpha phase in a three-cycle window centered at $500 \mathrm{~ms}$, then smoothed with a 30 -trial moving average ( $\mu \mathrm{V}$ as at $\mathrm{Oz}$ ). Weak partial phase resetting is indicated by 'joints' in the phase distribution wavefronts near $500 \mathrm{~ms}$. Lower traces: average ERP, ERSP and ITC time courses at the peak alpha frequency. Shaded areas show non-significant values $(p<0.01)$. Weak but significant phase resetting (in ITC) produces 'alpha ringing' in the ERP even during alpha power decrease (ERSP). Stronger potentials (red) near $500 \mathrm{~ms}$ reflect the slow positive offset and more precise phase sorting in this interval. Bottom: Dual-dipole model as in Figure 1. (b) Bilateral component exhibiting a marked ('true') ERP. From infomax decomposition of 450 71-channel trials during visual letter encoding. ERP-image trials phase-sorted at $9 \mathrm{~Hz}(\mu \mathrm{V}$ as at $\mathrm{O1})$. Inter-stimulus intervals were fixed, allowing pre-as well as post-stimulus phase-locking (ITC).

time clearly distinguish stimulus-locked and responselocked ERP features [31].

\section{Benefits of the new approach}

Clearly, 'evoked' and 'induced' event-related EEG dynamics are not mutually exclusive. Rather, eventrelated spectral dynamics might occupy all portions of the time/frequency state space (Figure 3), including regions without a current acronym in the EEG literature. Combining ICA preprocessing with time/frequency modeling has several benefits for cognitive neuroimaging and neuroscience. ICA minimizes the influence of volume conduction and identifies the activities of the individual cortical sources. Their oscillatory dynamics can be more physiologically plausible, more tightly linked to behavior, and amenable to trial-by-trial analysis than conventional ERP or ERSP measures applied to scalp data [5,28,31,39]. Possibly, the combined ICA time/frequency approach might prove to index brain pathophysiology more accurately than conventional measures. Ultimately, the new approach may help to bring non-invasive and invasive electrophysiological research into more direct relationship [42]. An open source toolbox, EEGLAB, implementing the analysis approach outlined here is freely available (www. sccn.ucsd.edu/eeglab; [35]).

\section{Possible pitfalls}

For some researchers accustomed to standard EEG analysis methods, the ICA time/frequency approach might amount to a perplexing paradigm shift. As with most new methods, some user cautions are in order. First, as a 'blind' separation technique, ICA cannot be guaranteed to yield physiologically meaningful results in each and every case. Not every independent component is equally robust statistically or equally plausible physiologically. The quality of an ICA decomposition can vary considerably with the suitability of the input data, and possibly with the ICA algorithm used. Second, not all 


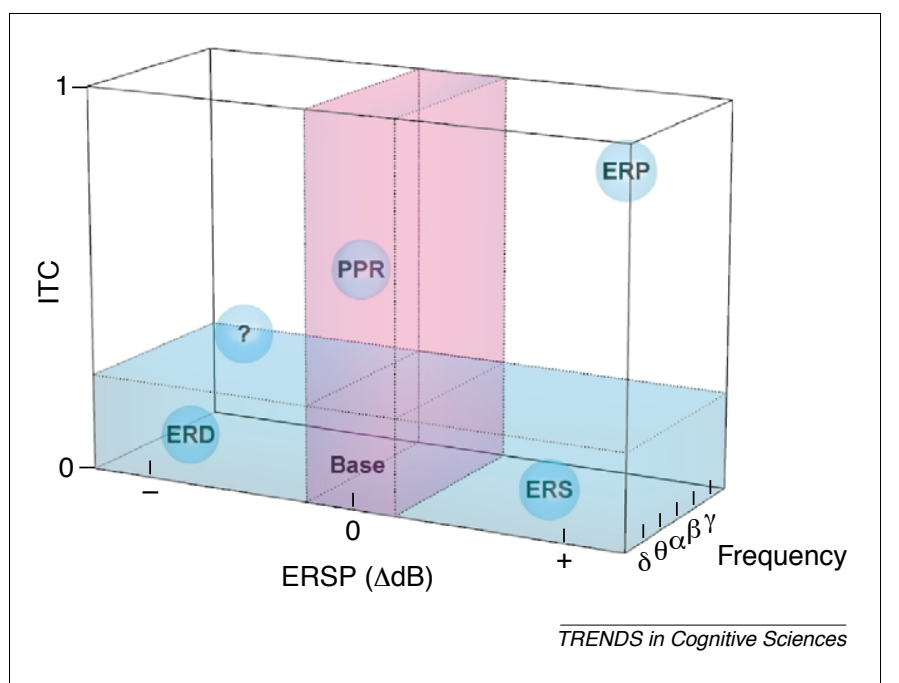

Figure 3. Event-related brain dynamic state space. State space of possible eventrelated dynamics of an EEG process at a given latency relative to experimental events. Depth axis: EEG frequency. Horizontal axis: mean change in spectral power from baseline (ERSP, in $\mathrm{dB}$ ). Vertical axis: phase locking of the activity to the time-locking events (ITC). Colored areas: regions of non-significant change from baseline state (Base). ERD: event-related desynchronization and synchronization (ERS) power changes, without phase-locking. PPR: partial phase resetting, without power change. ERP: evoked activity with strong phase-locking and power increase (see Figure 2b). (?): phase-locking during power decrease (Figure 2a).

frequency bands are treated equally by current ICA methods. In our experience, gamma-band and near-DC dynamics appear to be less well modeled than activity in intermediate frequency bands [43]. The application of time/frequency analysis to EEG data also involves inherent trade-offs and uncertainties that should be understood by researchers using these methods [35]. Finally, the same set of single-trial data, sorted by different parameters, can produce quite different ERP image representations. Care is required to understand how these appearances can co-exist and to avoid over-interpreting or misinterpreting their details.

\section{Future directions}

Certainly, further research will be required to fully evaluate the strengths, weaknesses and full implications of the new approach (see Box 2). However, the analysis model outlined here is still largely descriptive. Direct generative models of cortical dynamics involving realistic, multiscale finite-element modeling of field activity in neuropile (the most common usage) are a worthwhile

\section{Box 2. Questions for future research}

- Can the cortical extent of independent component (IC) sources be determined?

- Can ICA model near-DC and high-frequency gamma band dynamics?

- Which EEG/MEG phenomena do not fit the spatially fixed IC model?

- How similar are EEG source locations and dynamics across subjects and tasks?

- How do EEG sources compare with local field activities recorded invasively?

- In what ways can the time/frequency approach be usefully generalized?

- How can trial-to-trial and subject-to-subject variability in EEG dynamics best be characterized? goal. Current generative models [13,44-46] include many free parameters. It would be desirable to find ways to derive such models from actual data, using minimal assumptions, possibly by using still more flexible ICA approaches [43]. Methods for further characterizing trialto-trial variability and relating it to new and richer measures of behaviors might also prove valuable. However, cognitive neuroscientists with access to carefully recorded high-density EEG (and/or MEG) signals need not wait to mine more of the rich lode of information about event-related brain dynamics contained in their data.

\section{Acknowledgements}

Supported by The Swartz Foundation and the invaluable collaboration of Terrence J. Sejnowski and Anthony J. Bell at The Salk Institute and TzyyPing Jung at the Institute for Neural Computation, UCSD.

\section{References}

1 von Stein, A. et al. (2000) Top-down processing mediated by interareal synchronization. Proc. Natl. Acad. Sci. U. S. A. 97, 14748-14753

2 Fries, P. et al. (2001) Modulation of oscillatory neuronal synchronization by selective visual attention. Science $291,1560-1563$

3 Salinas, E. and Sejnowski, T.J. (2001) Correlated neuronal activity and the flow of neural information. Nat. Rev. Neurosci. 2, 539-550

4 Makeig, S. (1993) Auditory event-related dynamics of the EEG spectrum and effects of exposure to tones. Electroencephalogr. Clin. Neurophysiol. 86, 283-293

5 Makeig, S. et al. (2002) Dynamic brain sources of visual evoked responses. Science 295, 690-693

6 Stettler, D. et al. (2002) Lateral connectivity and contextual interactions in macaque primary visual cortex. Neuron $36,739-750$

7 Bagshaw, A. et al. (2003) Electrical impedance tomography of human brain function using reconstruction algorithms based on the finite element method. Neuroimage 20, 752-764

8 Wright, J.J. et al. (2001) Toward an integrated continuum model of cerebral dynamics: the cerebral rhythms, synchronous oscillation and cortical stability. Biosystems 63, 71-88

9 Freeman, W.J. and Barrie, J.M. (2000) Analysis of spatial patterns of phase in neocortical gamma EEGs in rabbit. J. Neurophysiol. 84, 1266-1278

10 Freeman, W.J. et al. (2003) Aperiodic phase re-setting in scalp EEG of beta-gamma oscillations by state transitions at alpha-theta rates. Hum. Brain Mapp. 19, 248-272

11 Suffczynski, P. et al. (2001) Computational model of thalamo-cortica networks: dynamical control of alpha rhythms in relation to focal attention. Int. J. Psychophysiol. 43, 25-40

12 Jones, S. et al. (2000) Alpha-frequency rhythms desynchronize over long cortical distances: a modeling study. J. Comput. Neurosci. 9, $271-291$

13 Suffczynskia, P. et al. (2000) Computational model of thalamo-cortical networks: dynamical control of alpha rhythms in relation to focal attention. Int. J. Psychophysiol. 43, 25-40

14 Engel, A.K. et al. (2001) Dynamic predictions: oscillations and synchrony in top-down processing. Nat. Rev. Neurosci. 2, 704-716

15 Hupe, J-M. et al. (2001) Feedback connections act onthe early part of the responses in monkey visual cortex. J. Neurophysiol. 85, 134-145

16 Sayers, B.M. and Beagley, H.A. (1974) Objective evaluation of auditory evoked EEG responses. Nature 251, 608-609

17 Fischer, Y. et al. (2002) Simultaneous activation of gamma and theta network oscillations in rat hippocampal slice cultures. J. Physiol. 539, $857-868$

18 Hajos, M. et al. (2003) Norepinephrine but not serotonin reuptake inhibitors enhance theta and gamma activity of the septo-hippocampal system. Neuropsychopharmacology 28, 857-864

19 Elbert, T. and Rockstroh, B. (1987) Threshold regulation: a key to the understanding of the combined dynamics of EEG and event-related potentials. J Psychophysiol. 4, 317-333

20 Tass, P.A. (1999) Phase Resetting in Medicine and Biology: Stochastic Modelling and Data Analysis, Springer-Verlag 
21 Badea, A. et al. (2003) Surface visualization of electromagnetic brain activity. J. Neurosci. Methods 127, 137-147

22 Gross, J. et al. (2003) Properties of MEG tomographic maps obtained with spatial filtering. Neuroreport 19, 1329-1336

23 Tass, P.A. et al. (2003) Synchronization tomography: a method for three-dimensional localization of phase synchronized neuronal populations in the human brain using magnetoencephalography. Phys. Rev. Lett. 90, 088101

24 Baillet, S. et al. (2001) Electromagnetic brain mapping. Signal Proc. Mag. IEEE 18, 14-30

25 Makeig, S. et al. (1996) Independent component analysis of electroencephalographic data. Adv. Neural Inf. Process. Syst. 8, 145-151

26 Jung, T-P. et al. (2001) Imaging brain dynamics using independent component analysis. Proc. IEEE 89, 1107-1122

27 Lee, T.W. et al. (2000) A unifying information-theoretic framework for independent component analysis. Comput. Math. Appl. 39, 1-21

28 Makeig, S. et al. (1997) Blind separation of auditory event-related brain responses into independent components. Proc. Natl. Acad. Sci. U. S. A. 94, 10979-10984

29 Bell, A.J. and Sejnowski, T.J. (1995) An information-maximization approach to blind separation and blind deconvolution. Neural Comput. $7,1129-1159$

30 Amari, S. (1998) Natural gradient works efficiently in learning. Neural Comput. 10, 251-276

31 Makeig, S. et al. (1999) Functionally independent components of the late positive event-related potential during visual spatial attention J. Neurosci. 19, 2665-2680

$32 \mathrm{Oja}$, E. and Hyvarinen, A. (2000) Independent component analysis: algorithms and applications. Neural Netw. 13, 411-430

33 Vorobyov, S. and Cichocki, A. (2002) Blind noise reduction for multisensory signals using ICA and subspace filtering, with application to EEG analysis. Biol. Cybern. 86, 293-303

34 Jung, T.P. et al. (2000) Removing electroencephalographic artifacts by blind source separation. Psychophysiology 37, 163-178

35 Delorme, A. and Makeig, S. (2004) EEGLAB: an open source toolbox for analysis of single-trial EEG dynamics including independent component analysis. J. Neurosci. Methods 134, 9-21
36 Onton, J. and Makeig, S. (2003) Distributed EEG dynamics of shortterm memory. In Cognitive Neuroscience Society (Abstr.), p. 217, JCN

37 Hunt, E. (1986) Mathematical models of the event-related potential. Psychophysiology 22, 395-403

38 Oostendorp, T.F. and van Oosterom, A. (1996) The surface Laplacian of the potential: theory and application. IEEE Trans. Biomed. Eng. 43, 394-405

39 Makeig, S. et al. (2000) A natural basis for brain-actuated control. IEEE Trans. Rehabil. Eng. 8, 208-211

40 Tallon-Baudry, C. et al. (1996) Stimulus specificity of phase-locked and non-phase-locked $40 \mathrm{~Hz}$ visual responses in human. J. Neurosci. 16 , $4240-4249$

41 Pfurtscheller, G. and Lopes da Silva, F. (1999) Event-related EEG/ MEG synchronization and desynchronization: basic principles. Clin. Neurophysiol. 110, 1842-1857

42 Makeig, S. (2002) Event-related brain dynamics - Unifying brain electrophysiology. Trends Neurosci. 25, 390

43 Anemueller, J. et al. (2003) Complex independent component analysis of frequency-domain electroencephalographic data. Neural Netw. 16, $1311-1323$

44 Freeman, W.J. (1975) Mass Action in the Nervous System, Academic Press

45 Chapman, C. et al. (2002) Spatial eigenmodes and synchronous oscillation: co-incidence detection in simulated cerebral cortex. J. Math. Biol. 45, 57-78

46 Rennie, C. et al. (2002) Unified neurophysical model of EEG spectra and evoked potentials. Biol. Cybern. 86, 457-471

47 Jutten, C. and Herault, J. (1991) Blind separation of sources: I. An adaptive algorithm based on neuromimetic architecture. Signal Process. 24, 1-10

48 Cardoso, J-F. and Souloumiac, A. (1993) Blind beamforming for non Gaussian signals. IEEE Proc. 140, 362-370

49 Vigario, R. et al. (2000) Independent component approach to the analysis of EEG and MEG recordings. IEEE Trans. Biomed. Eng. 47, $589-593$

50 Tang, A. et al. (2002) Independent components of magnetoencephalography: localization. Neural Comput. 14, 1827-1858

\section{Free journals for developing countries}

The WHO and six medical journal publishers have launched the Access to Research Initiative, which enables nearly 70 of the world's poorest countries to gain free access to biomedical literature through the Internet.

The science publishers, Blackwell, Elsevier, the Harcourt Worldwide STM group, Wolters Kluwer International Health and Science, Springer-Verlag and John Wiley, were approached by the WHO and the British Medical Journal in 2001. Initially, more than 1000 journals will be available for free or at significantly reduced prices to universities, medical schools, research and public institutions in developing countries. The second stage involves extending this initiative to institutions in other countries.

Gro Harlem Brundtland, director-general for the WHO, said that this initiative was 'perhaps the biggest step ever taken towards reducing the health information gap between rich and poor countries'. 